\title{
La lutte contre le crime organisé en Sicile. L'opération militaire Vespri Siciliani
}

\section{Anastassia Tsoukala}

\section{(2) OpenEdition}

1 Journals

Édition électronique

URL : http://journals.openedition.org/conflits/1615

DOI : 10.4000/conflits.1615

ISSN : $1777-5345$

Éditeur :

CCLS - Centre d'études sur les conflits lilberté et sécurité, L'Harmattan

Édition imprimée

Date de publication : 1 décembre 2004

Pagination : 51-61

ISBN : 2-7475-7598-X

ISSN : 1157-996X

Référence électronique

Anastassia Tsoukala, «La lutte contre le crime organisé en Sicile. L'opération militaire Vespri Siciliani », Cultures \& Conflits [En ligne], 56 | hiver 2004, mis en ligne le 07 janvier 2010, consulté le 30 mars 2021. URL : http://journals.openedition.org/conflits/1615; DOI : https://doi.org/10.4000/ conflits. 1615

Ce document a été généré automatiquement le 30 mars 2021.

Creative Commons License 


\title{
La lutte contre le crime organisé en Sicile. L'opération militaire Vespri Siciliani
}

\author{
Anastassia Tsoukala
}

1 Contrairement à d'autres pays européens, où l'émergence des militaires comme acteurs importants dans le maintien de la sécurité intérieure est un phénomène relativement récent, lié à la fin de la bipolarité, l'Italie n'a jamais établi de frontières nettement tracées entre la sécurité extérieure et la sécurité intérieure ${ }^{1}$ et l'usage actuel des militaires dans des missions relatives à la sécurité intérieure n'est guère perçu comme menaçant pour les structures démocratiques du pays. Cette légitimité dont jouissent les opérations militaires de ce type résulte d'une part d'une longue tradition en matière d'implication des forces armées dans des opérations de contrôle du territoire national et, d'autre part, de la constitution d'un solide cadre juridique réglementant celles-ci.

2 Ainsi, depuis la formation de l'Etat, en 1860, jusqu'à l'immédiat après-guerre, les forces armées étaient-elles directement intervenues tant dans la lutte contre le brigandage que dans la répression de révoltes, de mouvements populaires, de mouvements ouvriers, etc. Dans l'après-guerre, le gouvernement a progressivement limité ces interventions à des situations extraordinaires. Plus précisément, dans les années 1960, les forces armées ont été impliquées dans la lutte contre le terrorisme local à des fins séparatistes, dans la région du Haut Adige. Dans les années 1970, elles avaient été chargées d'opérations de maintien de l'ordre en Calabre, de la surveillance de la ligne ferroviaire Chiusi-Bologna ${ }^{2}$ et du contrôle du territoire suite à l'enlèvement d'Aldo Moro.

3 Mais, alors que, dans les années 1980, toutes les tâches liées à la sécurité intérieure étaient exclusivement confiées aux forces de police ${ }^{3}$, la fin de la bipolarité a marqué un tournant important en la matière, et les années 1990 ont été marquées par la réémergence des militaires comme acteurs essentiels pour le maintien de l'ordre et de la sécurité à l'intérieur du pays. Ceux-ci se trouvent en fait impliqués dans nombre de missions de surveillance et de contrôle du territoire $e^{4}$. Cependant, les opérations 
récentes se différencient de celles des décennies précédentes à maints égards. D’abord, quant à leur fréquence, puisque dans les années 1990 ont été menées onze opérations, contre quatre au cours des décennies 1970 et 1980. Ensuite, quant à leurs objectifs, puisqu'elles ne répondent plus uniquement à des situations ponctuelles mais visent aussi à combattre des comportements délinquants inscrits dans la durée, tels que la criminalité organisée et l'immigration clandestine, mettant de la sorte en lumière l'incapacité de l'Etat à gérer ces phénomènes en ayant recours uniquement aux forces de police. Après, quant à leur durée, qui est à présent nettement plus longue, atteignant souvent deux ans et, dans le cas de l'opération Vespri Siciliani, six ans. Enfin, quant au statut juridique du personnel militaire impliqué dans ces opérations, lequel, dès 1992, peut être investi du statut d'agent de sécurité publique.

4 Toutes ces modifications suggèrent une profonde transformation de la nature même de la participation des militaires dans des missions de sécurité intérieure. Loin de se limiter à des opérations ponctuelles et extraordinaires, cette participation tend progressivement à revêtir aussi un aspect ordinaire, comme nous l'indiquent par ailleurs les interventions quotidiennes de la marine dans le canal d'Otrante, qui concourt les forces de police afin d'empêcher la traversée nocturne du canal par des immigrés clandestins, ou l'usage régulier d'aéroports militaires pour l'exécution des mesures d'éloignement du territoire des immigrés clandestins.

Même si, actuellement, l'implication des forces armées dans la sécurité intérieure est de moins en moins insolite en Europe occidentale ${ }^{5}$, sa manifestation au niveau national soulève de nombreuses questions liées tant à ses fondements juridiques et aux modalités de la coopération inter-forces qu'à une certaine redéfinition du rôle des forces armées au sein de leur société. Les réponses avancées par l'Italie, lors de la plus importante des opérations menées dans les années quatre-vingt-dix, l'opération Vespri Siciliani, semblent donc être bien intéressantes, car non seulement elles nous présentent un cadre de réglementation bien élaboré, mais aussi elles mettent en lumière d'autres problèmes auxquels sont confrontés les militaires dans ce type de missions.

\section{Les objectifs de l'opération}

6 L'opération Vespri Siciliani a été menée en Sicile, de 1992 à 1998, afin de combattre les réseaux locaux de criminalité organisée. La mission assignée aux forces armées consistait à aider, par des actions de substitution ou de coopération, les forces de police en matière de contrôle du territoire et de surveillance d'objectifs sensibles, conformément aux directives données aux commandants militaires par les Préfets des régions concernées.

7 Cette opération visait formellement à permettre une meilleure redistribution des forces de police qui, dégagées des tâches secondaires, comme la surveillance d'objectifs sensibles, pourraient se consacrer davantage à leurs tâches de police judiciaire. Mais, à cet objectif formel s'ajoutaient implicitement deux objectifs majeurs, d'ordre symbolique. D'une part, l'opération visait à réaffirmer la présence de l'Etat, dont la crédibilité a été mise en jeu par la gravité des atteintes à la sécurité publique portées par la mafia sicilienne. Le recours aux forces armées était, à cet égard, particulièrement judicieux car, dans un pays souffrant toujours d'une forte fracture Nord/Sud, l'armée constituait le facteur de cohésion nationale par excellence. Ce souci de mettre en avant 
la cohésion et la solidarité nationale nous paraît d'ailleurs clairement illustré par le fait que la majorité des effectifs mobilisés venait du centre et du nord du pays. D'autre part, l'opération visait à rassurer la population sicilienne, en faisant baisser son sentiment d'insécurité vis-à-vis de la criminalité et, notamment, de la criminalité organisée.

La décision du gouvernement de l'époque d'avoir recours aux forces armées afin de mieux gérer ce phénomène criminel constituait, certes, une réponse aux activités de la puissante mafia locale, mais, en même temps, reflétait l'ordre hiérarchique des menaces alors établi au sein des agences de sécurité publique. En effet, tandis que dans nombre de pays européens le terme de nouvelle menace émergeant depuis la fin de la bipolarité était souvent lié à l'immigration clandestine, en Italie il était avant tout lié à la criminalité organisée transnationale, au blanchiment d'argent et à la mondialisation de l'économie criminelle. Malgré son ampleur, l'immigration clandestine n'était considérée comme une menace importante aux yeux des agents de sécurité publique que dans la mesure où elle était liée à des réseaux de criminalité organisée.

9 La volonté gouvernementale de mobiliser l'ensemble des agences de sécurité publique face à ce qui était considéré comme la plus importante des menaces pesant sur la sécurité du pays n'aurait pourtant pas trouvé un écho aussi favorable au sein des forces armées si elle n'avait pas coïncidé avec le souci de celles-ci de s'adapter aux nouvelles configurations de l'ère post-bipolaire - leur déploiement dans des missions de sécurité intérieure constituant alors une de leurs principales stratégies de compensation suite à la disparition de l'ennemi soviétique. Par conséquent, même si l'Etat Major italien considérait que, à part un éventuel concours technique, la lutte contre le crime organisé relevait en priorité des forces de police et de la DIA ${ }^{6}$, nombre de militaires estimaient, dès le début des années 1990, qu'ils devaient s'y impliquer à une échelle importante, notamment par le biais du renseignement et de l'aide au contrôle des populations. Il n'est donc guère étonnant que l'assignation à l'Armée de Terre de l'opération Vespri Siciliani ait été, dès le début, favorablement accueillie par les militaires.

\section{La réglementation juridique et son application}

L'importance accordée à cette opération se manifeste tout d'abord par le fait que celleci se trouve à l'origine d'une importante réforme du cadre juridique réglementant la participation des forces armées dans la sécurité intérieure. En effet, jusqu'alors, cette participation se fondait juridiquement sur la loi $n^{\circ} 382$ du 11 juillet 1978 qui, entre autres, assignait aux forces armées la tâche de la sauvegarde des institutions démocratiques.

11 Mais, lorsque, après les attentats mafieux qui ont provoqué la mort des juges Falcone et Borsallino, en 1992, il a été décidé d'impliquer les militaires dans la lutte contre la criminalité organisée en Sicile, il est devenu évident que la réussite de cette mission nécessiterait des compétences dépassant celles jusqu'alors accordées aux forces armées. Ces nouvelles compétences ont été fixées par le décret-loi $n^{\circ} 349$ du 25 juillet 1992, lequel est devenu la loi no 386 du 23 septembre 1992.

12 En vertu de cette loi, le personnel militaire pouvait dès lors agir en ayant les fonctions des agents de sécurité publique, sans pouvoir exercer pour autant les fonctions de police judiciaire. Ce statut a permis aux militaires de procéder à des contrôles d'identité, à des perquisitions et à des fouilles de personnes et de moyens de transport 
suspects, de saisir d'objets matériels et, dans des situations bien concrètes, d'effectuer même des arrestations. Les perquisitions et fouilles ainsi effectuées devaient être notifiées, au plus tard dans 48 heures, au Procureur de la République compétent.

En principe, le personnel militaire investi de ce statut pouvait opérer de manière autonome. Néanmoins, dans la pratique, les patrouilles étaient mixtes ${ }^{7}$ et commandées par le supérieur en hiérarchie des agents des forces de police concernés. Quant aux conditions d'engagement du feu, les militaires avaient les mêmes droits que les agents des forces de police, mais chaque corps avait ses propres armes ${ }^{8}$. Toutefois, alors que, en théorie, c'étaient les préfets des régions concernées qui devaient avaliser les règles d'engagement de feu, en réalité les décisions étaient prises par les officiers des forces armées engagées sur le terrain.

Ces ambiguïtés quant à l'application des dispositions juridiques se reflètent d'ailleurs très clairement dans les discours des agences de sécurité impliquées dans cette opération. Ainsi, les représentants des forces armées que nous avons rencontrés ont mis l'accent sur l'étendue de nouveaux pouvoirs qui leur avaient été conférés en vertu du décret-loi n³49 et, notamment, sur la possibilité d'opérer de manière autonome, tout en attirant notre attention sur le fait que, le personnel militaire ne connaissant aucune contrainte d'horaire, les opérations militaires étaient, par définition, plus étendues dans le temps que celles des forces de police. En revanche, l'image présentée par les représentants des forces de police rencontrés tendait à minimiser l'importance du rôle accordé aux forces militaires. Ainsi, la capacité du personnel militaire à opérer de manière autonome a été souvent contestée, nos interlocuteurs ayant insisté sur l'existence de patrouilles mixtes, et ses tâches ont été présentées comme réduites à la seule surveillance d'objectifs sensibles fixes, excluant de la sorte toute mobilité des unités militaires.

\section{La mise en œuvre de l'opération}

15 Bien que la durée de l'opération ait été inconnue au début, il a été précisé d'emblée que la participation des forces armées devait avoir une durée limitée. Initialement, il était prévu que l'opération, commencée le 28 juillet 1992, prendrait fin le 31 décembre de la même année. Finalement, sa durée a été successivement prolongée jusqu'au 30 juillet 1998, date à laquelle l'opération a définitivement pris fin.

Les 144000 hommes, mobilisés dans le cadre de cette opération, ont été chargés des tâches suivantes: a) la surveillance d'objectifs sensibles (aéroports, complexes industriels, tribunaux, préfectures, prisons...); b) la surveillance et le contrôle de portions d'infrastructures routières et ferroviaires ; c) la mise en place de barrages de contrôle routiers ; d) la mise en œuvre d'opérations de ratissage dans des zones rurales ; e) la mise en place des « cordons de sécurité » dans des zones urbaines, afin de faciliter le déroulement de certaines interventions policières ; f) la surveillance d'œuvres d'art. En revanche, le personnel militaire n'avait pas le droit de faire irruption ou d'effectuer un raid à l'intérieur d'édifices, ou d'effectuer des contrôles à l'intérieur d'édifices publics ouverts au public.

17 Les objectifs à surveiller étaient définis par les préfets qui, en concertation avec les commandants militaires concernés, fixaient le mode de déroulement des opérations tant à l'échelle régionale qu'à l'échelle locale. Les modalités exécutives étaient définies par les commandants des unités militaires locales, en concertation avec les 
commandants des forces de police concernées. Le bon déroulement des opérations était, en outre, assuré par un support informatique considérable.

Un des problèmes majeurs auxquels ont été confrontées les forces armées consistait à rendre compatible l'entraînement de leurs effectifs avec les particularités de la mission qui leur était dévolue, afin d'éviter tout risque d'accident ou de bavure. Ce problème était d'autant plus grave que les effectifs mobilisés étaient essentiellement composés de simples appelés, jeunes et inexpérimentés. L'adaptation aux exigences de ces nouvelles tâches a, donc, requis un entraînement ad hoc ainsi qu'une formation plus approfondie. Dans le premier cas, on a prévu un nombre considérable de leçons de tir et d'exercices diurnes et nocturnes en groupe. Dans le deuxième cas, on a planifié des exercices nécessitant, par exemple, l'exécution d'actions en cas d'absence de commandant, la prise de décisions en autonomie, la responsabilisation de tous les membres des unités concernées, et la préparation multifonctionnelle de celles-ci.

\section{L'évaluation de l'opération}

19 Dans le cadre de l'opération, ont été contrôlés 811766 personnes, dont 1171 ont été remises aux forces de police, ainsi que 660000 véhicules et 58000 édifices. En outre, ont été saisis 166 armes et plus de $3000 \mathrm{~kg}$ d'explosifs et de munitions. Ces chiffres impressionnants ne nous permettent toutefois pas d'évaluer aisément l'efficacité de Vespri Siciliani. Indéniablement, à la fin de l'opération, la mafia sicilienne se présentait considérablement affaiblie, car les autorités publiques avaient démantelé grand nombre de réseaux liés à la criminalité organisé en arrêtant plusieurs de leurs membres et même certains de leurs chefs. Mais, ces succès ayant été, en fait, largement dus aux aveux faits par des repentis et, sans doute, aux activités de la DIA, la contribution des militaires en la matière reste, malgré tout, imprécise. En outre, la portée de ces succès doit être relativisée par le fait que cette perte d'influence de la Cosa Nostra n'a pas impliqué l'affaiblissement des activités liées à la criminalité organisée en Italie mais plutôt leur déplacement au profit de la N'dragheta en Calabre, qui a renforcé sa position et étendu ses réseaux, en récupérant les trafics de la Cosa Nostra99.

En revanche, il semble incontestable que l'opération Vespri Siciliani ait provoqué une baisse de plus de $50 \%$ de la petite délinquance en Sicile, ce qui, à son tour, a fait baisser le sentiment d'insécurité de la population. Certes, cette baisse peut être de courte durée après le départ des effectifs militaires, mais l'apaisement de ce sentiment d'insécurité était un des principaux objectifs symboliques de l'opération.

21 Egalement réussie a été la réalisation du deuxième objectif symbolique, à savoir la réaffirmation de la présence de l'Etat, le déploiement des militaires ayant rassuré la population et confirmé la solidarité nationale, en renforçant de la sorte la confiance de la population aux institutions. Partagée par une grande partie de la population, par les médias et les autorités locales, cette perception de l'opération nous paraît bien révélée par les propos du maire de Palerme qui, considérant la présence de l'armée sur l'île comme la preuve de l'intérêt porté par l'Etat vis-à-vis de la population sicilienne, souhaitait, peu avant la fin de l'opération, soit la prolongation de celle-ci au-delà de 1998, soit le maintien permanent de certains effectifs militaires sur l'île pour qu'ils continuent à prêter concours, à échelle réduite, aux forces de police locales. 


\section{La réaction de la population} l'opération Forza Paris et d'autres opérations ultérieures, ait suscité d'importantes polémiques, en réveillant des craintes d'une militarisation de l'île, l'opération Vespri Siciliani a vite été favorablement accueillie par les médias et la population. Alors que, au début de l'opération, les médias se montraient sceptiques, en mettant en avant le danger de la militarisation du territoire et en mettant en cause la capacité des forces armées à gérer la criminalité organisée, au cours de l'opération et après sa fin il est devenu clair que l'opinion publique était clairement favorable à l'opération et que, en général, elle souhaitait le déroulement d'autres missions de ce type sur le territoire. Ceci résulte, certes, du fait que la criminalité organisée était perçue comme une grave menace pour la sécurité de l'ensemble du pays, dans la mesure où elle mettait potentiellement en jeu la stabilité même de l'Etat démocratique. Mais, en même temps, cet accueil favorable de l'opération a été le fruit d'une politique médiatique très élaborée de la part des forces armées, qui se sont efforcées de rendre clairs auprès de la population les motifs justifiant cette intervention militaire, afin de ne pas répéter les erreurs du passé. Il faudrait rappeler à cet égard que le développement de la mafia sicilienne est en grande partie dû à l'absence de légitimation auprès de la population sicilienne de la répression militaire du brigandage, au $19^{\text {ème }}$ siècle. Plus récemment, la répression militaire du terrorisme dans le Haut Adige avait suscité une telle hostilité de la part de la population locale qu'elle avait fini par mettre en jeu les relations diplomatiques entre l'Italie et certains pays limitrophes.

Souhaitant alors s'assurer d'avance du consensus de la population, les forces armées ont mis en place une politique médiatique articulée autour de trois axes : l'information, en vue de parvenir à gérer les médias plutôt qu'à être gérées par ceux-ci, la communication, en vue de créer des possibilités de rencontres entre le personnel militaire et la population locale ${ }^{10}$, et l'établissement d'un rapport interactif entre les deux premiers axes. Les efforts déployés à des fins médiatiques et les enjeux sousjacents ont été si importants que nous pourrions avancer que, à certains égards, l'opération Vespri Siciliani a été, avant tout, une opération de communication sociale. opération sur le terrain, ainsi que le fait qu'elle était la première opération menée par un personnel investi du statut d'agents de sécurité publique, ont érigé l'opération Vespri Siciliani au rang d'un véritable laboratoire pour de futures missions semblables.

En effet, l'opération a permis aux forces armées de fournir à leurs effectifs une formation réaliste, sur le terrain, sur des tâches qui pourraient faire partie intégrante de certaines de missions assignées aux militaires dans le futur. En outre, vu que les forces armées sont à présent appelées à reconfigurer leurs missions et modes d'intervention afin de faire face aux nouvelles menaces actuelles, liées à la gestion de crises plutôt qu'à des conflits impliquant d'ennemis externes ${ }^{11}$, le bon déroulement de l'opération a démontré que celles-ci disposaient d'une capacité d'adaptation importante et que, par conséquent, elles pouvaient répondre de manière efficace eux exigences posées par les nouvelles demandes de sécurité intérieure.

Ceci est d'autant plus possible que, les modalités opérationnelles des missions de maintien de la paix menées à l'étranger étant essentiellement semblables à celles des missions de sécurité intérieure menées sur le territoire national, les unes servent de 
modèle aux autres. Ainsi, l'expérience acquise lors de l'opération Vespri Siciliani et d'autres opérations du même type, servira, à son tour, aux futures missions éventuelles de maintien de la paix à l'étranger, et vice versa. Si cette interaction met bien en évidence les liens de plus en plus étroits entre le champ de la sécurité intérieure et celui de la sécurité extérieure, elle permet également d'apaiser les craintes exprimées par des militaires directement impliqués dans l'opération Vespri Siciliani, qui ne souhaitaient guère voir les forces armées aplaties dans la dimension d'une force de police complémentaire dotée, par ailleurs, de pouvoirs limités.

Extraordinaire dans sa durée ${ }^{12}$, l'opération Vespri Siciliani l'est beaucoup moins dans la tendance actuelle qu'elle illustre de recoupement de l'ordre étatique interne avec l'ordre international, tel qu'il se manifeste par l'interpénétration des agences de sécurité intérieure et extérieure au sein de ce que certains chercheurs ont déjà qualifié de champ de la sécurité ${ }^{13}$. Nous indiquant clairement les modalités et les difficultés de la mise en œuvre d'une stratégie de policiarisation des fonctions militaires de combat ${ }^{14}$, elle représente une mise en cause de la capacité de l'Etat à assurer la surveillance et le contrôle de son territoire à travers ses seules forces de police, nous révélant par là non seulement la perméabilité des concepts de l'intérieur et de l'extérieur ${ }^{15}$, mais aussi la propre dynamique du processus de reconfiguration constante des éléments constitutifs du champ de la sécurité dans les pays occidentaux.

\section{NOTES}

1. Sur l'évolution du concept de sécurité en Italie depuis la fin de la Seconde Guerre Mondiale jusqu'à la fin de la bipolarité, voir les travaux de Jean C., Studi strategici, Franco Angeli, Milano, 1990, et Il Ministero Della Difesa, La Nuova Italia Scientifica, Roma, 1991, p. 27 et suivantes. En ce qui concerne la période post-bipolaire, voir Merlini C., «European Security from the Italian Perspective ", The International Spectator, avril-juin 1996, n², pp. 61-73.

2. Il s'agissait de la surveillance d'objectifs sensibles (ponts, viaducs, galeries...).

3. Ce terme désigne notamment la Polizia di Stato (police d'Etat), l'Arma dei Carabinieri (Arme des Carabiniers) et la Guardia di Finanza (Garde des Finances).

4. En 1990, les forces miliaires ont prêté leur concours logistique aux forces de police à l'occasion de la Coupe du Monde de football. En 1991, elles sont chargées de la surveillance d'installations et d'objectifs sensibles pendant la Guerre du Golfe, ainsi que du contrôle de plus de 20000 réfugiés albanais arrivés à Bari et hébergés pendant plus d'un an dans des locaux appartenant à l'armée de Terre. En 1992, elles sont chargées de l'opération Forza Paris en Sardaigne, qui consiste en un contrôle du territoire afin de lutter contre les réseaux locaux de criminalité organisée. En 1992, a également commencé l'opération Vespri Siciliani, qui a pris fin en 1998. De 1993 à 1995, les forces militaires prêtent leur concours à la préfecture d'Ancône, en assumant la surveillance de la raffinerie API et de l'aéroport civil de Falconara afin de prévenir tout acte terroriste et/ou de sabotage de la part des factions en guerre en ex-Yougoslavie. Pendant la même période, elles ont aussi mené l'opération Testuggine à Friuli, laquelle consistait en la surveillance de la frontière italo-slovène afin de lutter contre l'immigration clandestine, le trafic d'armes et le trafic de stupéfiants. De 1994 à 1995, elles ont mené les opérations Riace en Calabre et Partenope à Naples, 
consistant en des missions de contrôle du territoire et de surveillance d'objectifs sensibles. En 1995, elles ont aussi mené l'opération Salento aux Pouilles, assurant le contrôle de la frontière maritime des provinces des Pouilles afin de lutter contre l'immigration clandestine. Enfin, de 1997 à 1998, elles ont été chargées de l'opération Partenope 2 à Naples, consistant en des missions de contrôle du territoire et de surveillance d'objectifs sensibles.

5. Sur ce point, voir Bigo D., Rapport final sur la sécurité intérieure. Implications pour la Défense, 97/1057.A.000 DSP/DAS, Paris, 1998 ; Bigo D., Hanon J.-P., Tsoukala A., Approche comparative des problèmes de sécurité intérieure. Rapport final, 98/010.T/DSF/DAS, Paris, 1999.

6. Direzione Investigativa Antimafia (Direction des Investigations Antimafia).

7. Deux militaires pour un agent des forces de police.

8. Dans tous les cas, il s'agissait d'armes légères.

9. Bigo D., Tsoukala A., Bonelli L. et al., La fonction de protection. Une approche dynamique et dialectique, 99/027.T/DSF/DAS, Paris, 2001.

10. Celles-ci pouvaient prendre la forme de concerts, de tournois sportifs, de visites culturelles...

11. Sur le rôle des forces armées dans la gestion de conflits de basse intensité et la politique étrangère de l'Italie, voir Andreatta B., «L'Italia e l'Europa centrale e sud-orientale ", Affari esteri, janvier 1998, n 117, pp. 5-13.

12. Cette remarque ne devrait pas occulter la très longue durée de certaines opérations militaires de sécurité intérieure menées dans d'autres pays européens, comme le plan Vigipirate en France. 13. Bigo D., «La mondialisation de la sécurité ? Réflexions sur le champ des professionnels de la gestion des inquiétudes à l'échelle transatlantique et sur ses implications ", in Fortman M., Macleod A., Roussel S., Vers des périmètres de sécurité? La gestion des espaces continentaux en Amérique du Nord et en Europe, Montréal, Athéna, 2003 et Traces: a multilingual series of cultural theory No. 4 « Sovereign Police, Global Complicity », ed. Jon Solomon, Naoki Sakai, University of Hong Kong, 2004.

14. Cette stratégie est allée de pair avec une stratégie opposée mais en même temps complémentaire, à savoir l'internationalisation des missions de police.

15. Walker R., Inside/Outside : International Relations as Political Theory, Cambridge University Press, Cambridge, 1993.

\section{RÉSUMÉS}

Contrairement à d'autres pays européens, l'Italie n'a jamais établi de frontières nettement tracées entre sécurité extérieure et sécurité intérieure. L'étude de la mise en oeuvre de l'opération de lutte contre la criminalité organisée Vespri Siciliani (1992-1998) nous permet d'aborder nombre de questions liées aux fondements juridiques et aux modalités de coopération interforces, ainsi qu'à la redéfinition du rôle des forces armées dans les sociétés occidentales contemporaines.

Unlike most European countries, Italy has never established a clear boundary line between external and internal security. The study of the implementation of the counter-organized crime operation Vespri Siciliani (1992-1998) highlights several issues related to the legal ground and the modes of cooperation between security agencies as well as to the redefinition of the role held by the Army in contemporary Western societies. 
INDEX

Mots-clés : Italie, sécurité intérieure, coopération interforces, lutte contre la criminalité organisée

\section{AUTEUR}

\section{ANASTASSIA TSOUKALA}

Anastassia TSOUKALA est maître de conférences à l'université Paris -Sud XI, chercheur associé au Centre d'Etudes sur les Conflits et Senior Researcherdans les programmes ELISE et CHALLENGE. 